\section{Quantification of morphology of canine circumanal gland tumors: a fractal based study}

\author{
I.C. Šoštarić-Zuckermann, ${ }^{1}$ K. Severin, ${ }^{2}$ \\ M. Huzak, ${ }^{3}$ M. Hohšteter, \\ A. Gudan Kurilj,' B. Artuković, ${ }^{1}$ \\ A. Džaja, ${ }^{4} \check{Z Z}_{\text {. Grabarević }}$ \\ 1Department of Veterinary Pathology, \\ Veterinary Faculty, University of Zagreb \\ 2Department of Forensic Veterinary \\ Medicine, Veterinary Faculty Zagreb, \\ University of Zagreb \\ ${ }^{3}$ Department of mathematics, Faculty of \\ Science, University of Zagreb \\ ${ }^{4}$ M-SAN EKO d.o.o., Zagreb, Croatia
}

\section{Abstract}

Circumanal gland tumors are very common neoplasms of dogs. Their classification relies on microscopic examination and is further supported by a few immunohistochemical markers that help indicate their prognosis. However, new additional tests would be highly useful. The purpose of this study was to develop such a test using fractal analysis which is increasingly being applied in science, especially in the field of biomedicine. A total of 53 circumanal gland tumors were chosen from our department archives. After a precise histological classification according to the World Health Organization classification, the number of de novo classified samples was as follows: 15 adenomas, 11 epitheliomas, 21 well differentiated carcinomas, 6 poorly differentiated carcinomas. Ten samples of normal circumanal gland were also included as control. All samples were immunohistochemicaly stained with vimentin. All immunohistochemical reactions were photographed at two different magnifications -100X and 400X- and converted to 1 bit in black and white (bitmap) images, thus enhancing the positive vimentin reactions. These images were used for the assessment of fractal dimension applying the box counting method and computer software Fractalyse. To determine the significance of results, conventional statistics were performed using Statistica software.

The overall vimentin stain score was significantly higher in epitheliomas and carcinomas than in normal circumanal glands (CG) or adenomas. Mean values of fractal dimension estimated at magnification $100 \mathrm{X}$ and $400 \mathrm{X}$ were as follows: normal CG 1.318 and 1.372, CG adenomas 1.384 and 1.408, CG epitheliomas 1.547 and 1.597, CG well differentiated carcinomas 1.569 and 1.607 , CG poorly differentiated carci- nomas 1.679 and 1.723. Significant differences (at level of 5\%) of these values were observed between individual groups of CG adenomas or normal CG, and epitheliomas or carcinomas.

The above results indicate vimentin immunohistochemistry staining and assessment of fractal dimension as an ancillary diagnostic method of choice when discerning between benign and malignant tumors of circumanal glands. Additional development of the method of fractal dimension assessment may yield a possibility for this tool to successfully discern between all of the types of CG tumors.

\section{Introduction}

Circumanal gland (CG) tumors are the most common epithelial skin tumors in dogs, and the third most common of all canine skin tumors. ${ }^{1}$ They typically arise as nodular outgrowths in the perianal region of aged dogs. ${ }^{2}$ According to their behavior and histological appearance these tumors are classified into the more frequent benign adenomas, and the less frequent low grade-malignant epitheliomas and malignant carcinomas. ${ }^{3}$ Definite diagnosis of these neoplasms requires histopathologic examination which according to established criteria discerns between these and helps predict biological behavior. However, as with many neoplasms, there remains substantial interobserver variation when classifying these tumors. This is especially true for borderline cases. In such cases, additional tests prove to be beneficial. There have only been a few studied additional diagnostic/prognostic tools for these tumors (silver-stained nucleolar organizer regions $\mathrm{AgNOR}^{4}$ and Ki675), both cellular proliferation markers. Another ancillary diagnostic/prognostic tool, taking a different (non-proliferative) approach, would be a nice supplement to the whole diagnostic work up. Such a tool would be even more appreciated nowadays with the recent introduction of subclassification of circumanal gland carcinomas, which discerns between well differentiated and poorly differentiated carcinomas. ${ }^{2}$

Fractal analysis of images has proved as a valuable tool in various fields of medicine. Notable examples include common diagnostic imaging methods: electroencephalography, brain imaging, mammography and bone imaging. ${ }^{6}$ It has also proved as a very promising tool when harnessed to analyze oncologic microscopic samples. These could be hematoxylin and eosin (H\&E) stained slides ${ }^{7-10}$ or cytological specimens. ${ }^{11,12}$ Finally, in recent years, a few approaches combining specific immunohistochemistry (IHC) stains and fractal dimension (FD) calculating algorithms have been
Correspondence: Ivan-Conrado ŠoštarićZuckermann, Department of Veterinary Pathology, Veterinary Faculty, University of Zagreb, Heinzelova 55, 10000 Zagreb, Croatia. Tel. +385.1.2390312 - Fax: +385.1.2441390.

E-mail: isostaric@vef.hr

Key words: Circumanal gland; circumanal gland tumors; fractal; dog; vimentin; immunohistochemistry.

Contributions: ICŠZ, participation in study design, histopathological, IHC and fractal analysis, IHC evaluation, manuscript drafting; $\mathrm{KS}, \mathrm{AD}$, statistical and image analysis; $\mathrm{MHu}, \mathrm{BA}, \mathrm{Z} \mathrm{G}$, participation in study design, statistical analysis; MHo, AGK, histopathological analysis. All authors read and approved the final manuscript.

Funding: this study was supported by a project of the Ministry of Science and Technology, Republic of Croatia: Comparative diagnostic, morphometry, and analysis of human and animal tumors (053-05322642260, principal investigator: prof. dr. sc. Željko Grabarević).

Received for publication: 17 December 2015. Accepted for publication: 1 March 2016.

This work is licensed under a Creative Commons Attribution-NonCommercial 4.0 International License (CC BY-NC 4.0).

(C) Copyright I.C. Šoštarić-Zuckermann et al., 2016 Licensee PAGEPress, Italy

European Journal of Histochemistry 2016; 60:2609 doi:10.4081/ejh.2016.2609

elaborated to help diagnose and predict the behavior of certain types of human neoplasms. For instance, determining the fractal dimension of pancytokeratin stained sections of ductal carcinomas proved as a good indicator of malignancy. ${ }^{13,14}$ This same approach (using pancytokeratin) proved as a good tool for differentiation between non-small cell lung adenocarcinoma and squamous cell carcinoma. ${ }^{15} \mathrm{~A}$ cytokeratin stain was also used in FD determination of canine trichoblastomas, ${ }^{16}$ this being one of the rare examples of applying this method within veterinary medicine. There is an even larger number of studies using FD as a tool for assessing neoplastic (micro)vascularity. ${ }^{17-22}$ These studies usually include a CD34 stain for endothelial cells and a box-counting method of calculating FD. Most of these reports show promising results, indicating that such methods could help differentiate between various tumor subtypes, or give a more accurate prognosis for specific cases. ${ }^{17-21}$

Considering all of the above, we evolved a similar method combining vimentin IHC staining and fractal image analysis on tissues of normal and neoplastic canine circumanal glands. The decision to use vimentin staining 
was made after a successful pilot study which highlighted the pattern of fibrovascular stroma, but also reserve (basaloid cells) of the adenomatous and carcinomatous circumanal gland. ${ }^{23}$ The overall intention for this method was to be useful in the classification of $\mathrm{CG}$ tumors and eventually prognosis, but also to be simple and inexpensive.

\section{Materials and Methods}

\section{Samples}

Samples included in this study consisted of archive paraffin blocks from 43 excised tumor samples submitted to our department from January $1^{\text {st }}, 2006$ until December $31^{\text {st }}, 2010$, and originally diagnosed as CG epithelioma or carcinoma. To this number, ten CG adenomas from the same period and ten normal (non tumorous) CG samples were added giving a total of 63 samples. Normal circumanal gland samples were obtained from carcasses of dogs younger than four years submitted for a routine necropsy. These dogs had no visible pathologic process of CG, no clinical history of any CG ailment, and most of them succumbed to trauma or viral diseases.

\section{Microscopic examination and classification}

Standard H\&E slides were prepared from all of the selected samples (paraffin blocks). A microscopic examination was performed using guidelines presented in textbook, ${ }^{2}$ which distinguishes between well and poorly differentiated CG carcinoma and is otherwise consistent with the WHO classification of tumors. To lessen individual personal bias when making the histological diagnosis, this examination was conducted independently by three different veterinary pathologists. In case of individual conflicting diagnoses, a final diagnosis was made by consultation of all three pathologists.

\section{Immunohistochemical analysis}

A 4- $\mu$ m thick section of each chosen paraffin block sample was mounted on coated glass slides (Dako A/S, Glostrup, Denmark, K8020), dewaxed, and rehydrated. Antigen retrieval was performed with a citrate buffer $(\mathrm{pH} 6.0)$ in a microwave oven for $20 \mathrm{~min}$. Endogenous peroxidase activity was blocked by incubating the sections in Dako REAL ${ }^{\text {TM }}$ Peroxidase-Blocking solution for $5 \mathrm{~min}$. Sections were then incubated with the primary antibody - Monoclonal mouse anti-vimentin (Clone V9, M0725, Dako) diluted 1:100 for $30 \mathrm{~min}$. This was followed by incubation for 30 min with a ready-to-use secondary antibody (Dako REAL ${ }^{\mathrm{TM}}$ EnVision $^{\mathrm{TM}}$ / Horseradish Peroxidase, Rabbit/ Mouse) and with the substrate Dako REAL ${ }^{\mathrm{TM}}$
Diaminobenzidine + Chromogen for a further 10 min. Rinses were done with DakoCytomation Wash Buffer between each step. All of the previous steps (from antigen retrieval) were done in a Dako Autostainer. Finally, the sections were counterstained with hematoxylin, dehydrated and mounted with cover slips. A sample of connective tissue was used as a positive control, while negative controls were obtained by substitution of the primary antibody with phosphate buffered saline.

\section{Scoring of vimentin stained sections}

The IHC staining was scored using a semiquantitative method based on both intensity and proportion of cells stained, as already applied for this antibody, ${ }^{24}$ which allowed scores from 0 to 12 .

\section{Calculation of the fractal dimension}

Image (micrograph) acquisition and analysis

For each of the 64 IHC microscopic samples, three micrographs were taken using $100 \mathrm{X}$ magnification (microscope objective magnification 10X multiplied by standard ocular magnification of another 10X). Another three micrographs were taken using $400 \mathrm{X}$ magnification (microscope objective magnification 40X multiplied by standard ocular magnification of 10X). All images were captured using an Eclipse Nikon E600 microscope with installed Olympus DP20 camera and a computer software Cell B (Olympus Soft Imaging Solutions), producing a 1600x1200 pixel image (300 dpi) at both magnifications. Microscopic fields of view which were photographed were chosen at random, but within the part of the slide with evident immunohistochemical reaction. Also, parts of the slide which were not of adequate quality, and parts of the slide where dominant structures were non tumorous (in case of circumanal gland adenomas, epitheliomas, well and poorly differentiated carcinoma) or non glandular (in case of normal circumanal glands) were avoided. All of the micrographs were edited using Adobe Photoshop version CS6 (Adobe Systems, Mountain View, CA, USA), as follows.

First, the micrographs were converted to CMYK mode (cyan, magenta, yellow, black). In this color range (determined by CMYK mode) all of the non-yellow colors were removed, while at the same time enhancing the yellow color using option Image $>$ Adjustment $>$ Color balance (value: 100). In this way, the yellow (and brown) color range was enhanced and other colors were removed, thus enhancing the IHC reaction. The micrographs were further converted to 8 bit grayscale pictures (mode) which were additionally adjusted according to the distribution of pixels by light color tone range (using Image $>$ Adjustment $>$ Levels; values of tones: dark 0 , grey 0.01 and white 160 ). Finally, the pictures were converted to $1 \mathrm{bit}$ black and white bitmap type, which supports strictly black and white pictures without shades of gray.

This way, the black color represented positive (brown) labeling of the original IHC reactions of the vimentin. Examples of images from every described step of the process are presented in Figure 1. The pictures in bitmap type were suitable for estimation of the fractal dimension as described below.

\section{Assessment of the fractal dimension of vimentin positive signals}

The fractal dimension of vimentin positive signals was estimated using a box-counting algorithm integrated within the computer program Fractalyse 2.4. This software was set to estimate FD using the box counting method and grid algorithm with exponential increase of box size. This program counts boxes (squares) on a grid which contains one or more black marked pixels. With each step the size of the mentioned box is increased exponentially (base two); in the first step one box is equal to the size of one pixel. Each step of this process therefore yields one value $r$ which represents the side length of the box and associated value $n=n(r)$, which stands for the number of boxes with at least one positive value (signal). By repeating the mentioned steps a sequence of values $r$ and $n(r)$ is acquired. These values are amenable for placing on Cartesian coordinating system, where the ordinate ( $Y$ axis) represents the number of boxes (squares) with positive signals $(n(r))$, and the abscissa ( $X$ axis) represents side length ( $r$ ) of the corresponding box (square). Such an acquired curve is an empirical curve and has to be paired with an estimated curve by the formula:

since

$$
n(r) \approx r^{-\mathrm{FD}}
$$

$$
\mathrm{FD}={ }_{\lim r \rightarrow 0 \log }[1 / \mathrm{n}(\mathrm{r})] / \log \mathrm{r}
$$

by definition of the box-counting fractal dimension (FD). ${ }^{25}$

The value of the fractal dimension A FD is estimated by a linear regression upon an empirical log-log curve: ${ }^{26}$

$$
\log [1 / n(\mathrm{r})] \approx \mathrm{FD} \cdot \log \mathrm{r}
$$

A value of FD was estimated for each of the micrographs. Since for each sample three micrographs at magnification $100 \mathrm{X}$ and $400 \mathrm{X}$ were taken, this yielded three values of FD for each magnification. The mean standard deviations of these three measurements for each 
magnification were 0.062 (FD 100X) and 0.057 (FD 400X). Therefore, the fractal dimension of each sample was expressed by two values FD100X and FD400X, which represented the mean of the estimated FD for each micrograph at these two magnifications.

\section{Statistical analysis}

All data were analyzed using data analyzing software Statistica ver. 10.0. We used histological diagnosis as an ordinal variable since we had five different diagnoses from the same tissue that could be easily arranged according to its biological behavior. The diagnoses were therefore listed in the following order with increasing malignant behavior: 1, normal CG; 2, CG adenoma; 3, CG epithelioma; 4, well differentiated CG carcinoma; 5 , poorly differentiated CG carcinoma. Chi-Square test for homogeneity (see Test 16a in Sheskin ${ }^{27}$ ) was used to test the differences in the distributions (homogeneity) of vimentin scores among subpopulations (groups) of tumors classified with respect to histological diagnosis. One-way ANOVA (see Test 21 in Sheskin ${ }^{27}$ ) and Tukey's HSD test (see Test 21c in Sheskin ${ }^{27}$ ) were used to test differences in the means of FD among all, and between any two of the tumor subpopulations classified with respect to histological diagnosis. Lilliefors' corrected KolmogorovSmirnov's test and Shapiro-Wilk's test ${ }^{28}$ were used for testing normality of all individual groups of FD data. Bartlett's test (see Ch. 3-3.4 in Montgomery ${ }^{29}$ ) was used for testing homogeneity of variances in FD data among subpopulations classified with respect to the histological diagnosis. Normal probability plot $^{28}$ was used for graphical testing of normality of errors in ANOVA model. Goodman and Kruskal's gamma statistics (see Test 32 in Sheskin $^{27}$ ) was used to express the level of correlation between histological diagnosis and vimentin scores meant as two ordinal variables. The Spearman's rank correlation coefficient (see Test 29 in Sheskin ${ }^{27}$ ) was used to express correlation between histological diagnosis and FD, and vimentine scores and FD, as a measure of monotonic association between two ordinal variables. The Pearson's productmoment correlation coefficient (see Test 28 in
Sheskin $^{27}$ ) was used for measuring the level of linear association between vimentine scores (meant as numerical variable with equally distant and scaled values in a way that it can be interpreted as an interval/rational variable) and FD (obviously interval/rational variable).

\section{Results}

\section{Microscopic examination and clas- sification}

The microscopic examination of 43 samples originally diagnosed as CG carcinomas or epitheliomas revealed 21 well differentiated carcinomas, 11 epitheliomas, six poorly differentiated carcinomas and five adenomas. The five adenomas were further grouped with the previously selected group of ten CG adenomas. The total and final number of samples by diagnosis are presented in Table 1.

\section{Vimentin scores of IHC stained sec- tions}

A positive IHC reaction to vimentin was detected in the cytoplasm of reserve or hepatoid cells of normal circumanal glands or tumors. As expected, a positive reaction was also observed in connective tissue stroma. Staining intensity varied from a weak to a strong reaction, and there were also samples without any reaction. Generally speaking, staining intensity was more pronounced in malignant subtypes of tumors. The percentage of positive cells varied, and was also higher in
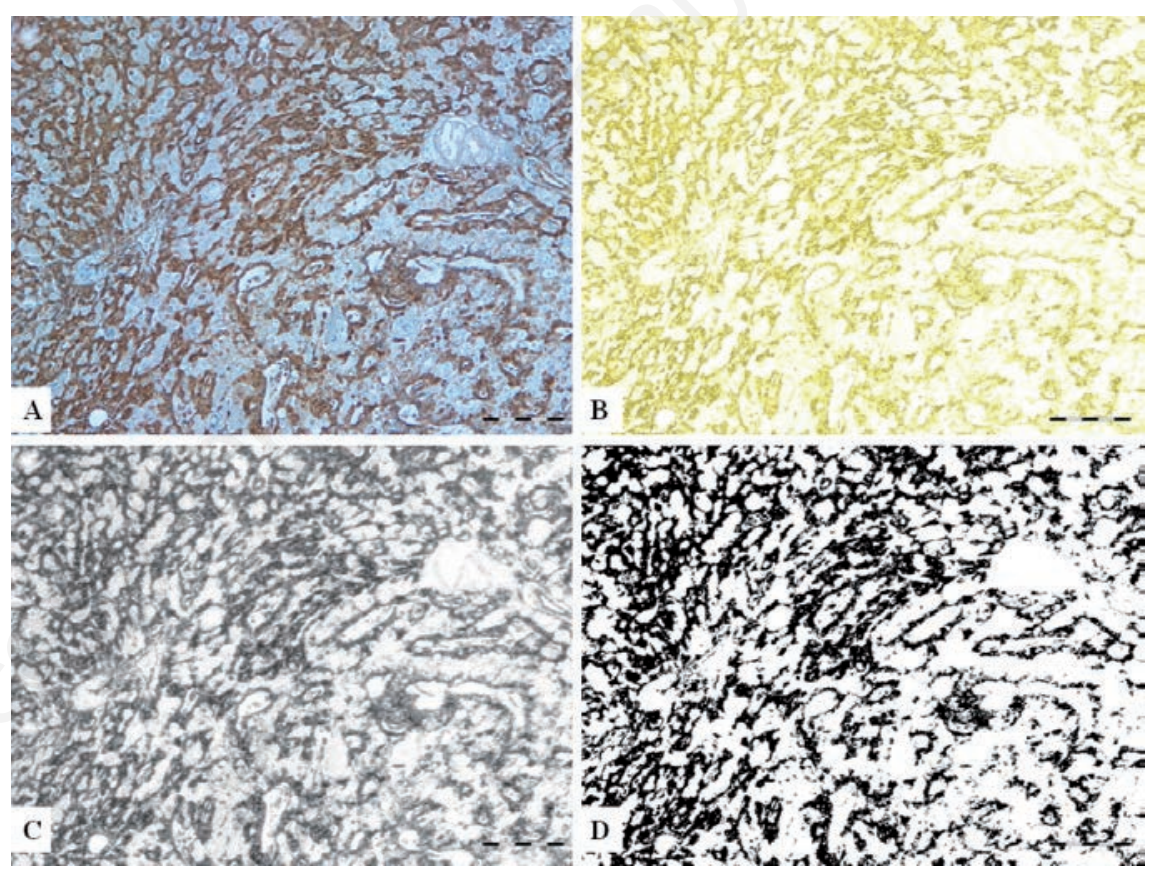

Figure 1. Steps in image transformation, yielding bitmap image from original IHC vimentin stained section micrograph. A) IHC vimentin stained section micrograph (for this illustration one well differentiated carcinoma is depicted). B) CMYK mode of image. C) Greyscale image. D) Final bitmap image suitable for fractal analysis. Scale bars: $200 \mu \mathrm{m}$.

Table 1. Samples by diagnosis (samples included in the study). Number of samples Mean age of dogs (in years) Sex ratio (M/F) Most common breed

\begin{tabular}{lccccc} 
Normal circumanal glands & 10 & 2.37 & $6 / 4$ & Mixed breed (4) \\
Circumanal gland adenomas & 15 & 8.13 & $12 / 3$ & Mixed breed (4) \\
\hline Circumanal gland epitheliomas & 11 & 10.91 & $11 / 0$ & Mixed breed (5) \\
Well differentiated circumanal gland carcinomas & 21 & 11.25 & $21 / 0$ & Poodle (3) \\
\hline Poorly differentiated circumanal gland carcinomas & 6 & 11.67 & $5 / 1$ & Mixed breed (2) Poodle (2) \\
Overall number of samples & 63 & 9.06 & $55 / 8$ & Mixed breed (17) \\
\hline
\end{tabular}


malignant types of tumors. The same was true for the final vimentin score, since it represented a product of percentage of the positive cells and their staining intensity. The median values of vimentin scores were as follows: 1 , normal CG; 2.5, CG adenomas; 3.5, CG epitheliomas; 2.5, CG well differentiated carcinomas; 6, CG poorly differentiated carcinomas.

Considering the relatively small number of samples and type of variable this score represented, we were obliged to consolidate these results as presented in Table 2 . This consolidation rendered this data suitable for a Chi square test, and Goodman and Kruskal's gamma statistics. The results of the Chi square test revealed that the difference between these two groups (normal CG and CG adenomas versus $\mathrm{CG}$ epitheliomas and carcinomas) was significant $\left(\mathrm{P}=5.7310^{-6}\right)$. Goodman and Kruskal's gamma rank correlation between such consolidated histological groups and vimentin scores was 0.898 .

\section{Fractal dimension of vimentin stained sections}

Fractal dimension was assessed at magnification 100X (FD100X) and 400X (FD400X). Mean values of FD100X and FD400X varied across different histological groups, and were as follows (95\% confidence interval limits

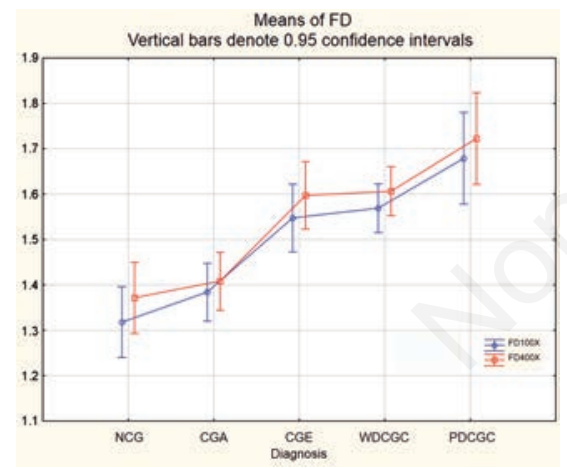

Figure 2. Mean values of fractal dimension for normal circumanal glands and different circumanal glands tumor types. The $\mathrm{Y}$ axis represents values of fractal dimension from 1.1 to 1.9 . The blue plot shows value of the fractal dimension assessed from IHC vimentin micrographs taken at magnification 100X, while the red plot shows value of the fractal dimension assessed from IHC vimentin micrographs taken at magnification 400X. NCG, normal circumanal glands; CGA, circumanal gland adenomas; CGE, circumanal gland epitheliomas; WDCGC, well differentiated circumanal gland carcinomas; PDCGC, poorly differentiated circumanal gland carcinomas. shown inside brackets): normal CG 1.318 $( \pm 0.0578)$ and $1.372( \pm 0.061), \mathrm{CG}$ adenomas $1.384( \pm 0.065)$ and $1.408( \pm 0.073)$, CG epitheliomas $1.547( \pm 0.101)$ and $1.597( \pm 0.092), \mathrm{CG}$ well differentiated carcinomas $1.569( \pm 0.052)$ and $1.607( \pm 0.051)$, CG poorly differentiated carcinomas $1.679 \quad( \pm 0.056)$ and 1.723 $( \pm 0.039)$. This data is graphically depicted in Figure 2, and also provided in Table 3 , where the lowest and highest recorded values of FD100X and FD400X and the corresponding values of standard errors, standard deviations and the mean measurement standard deviations are also given. Normality tests for each of these data groups showed that we were not able to rule out easily the normality assumption about the FD data (Table 3). Moreover Bartlett's test showed too that we were not able to rule out the assumption that their population variances are all equal (Bartlett's statistic are equal 5.77 and 7.71 providing P-values 0.22 and 0.10 at 4 degrees of freedom to FD $100 \mathrm{X}$ and FD 400X, respectively). Hence it seemed that applying one-way ANOVA to FD 100X and FD 400X with respect to the histological diagnosis (as categorical variable in this case) was appropriate. A one-way analysis of variance for testing equality of mean values of FD100X of individual groups of normal CG and neoplastic CG gave a P-value of $5.482 \cdot 10^{-8}(F$-statistic $=13.84$ at (4.58) degrees of freedom, sum of squares with respect to the histological diagnosis $=0.8456$, total sum of squres $=1.7313$ ). Analogue $p$ value for FD400X was $3.75010^{-8}(F$ statistic $=14.23$ at $(4,58)$ degrees of freedom, sum of squares with respect to histological diagnosis $=0.8693$, total sum of squares $=$ 1.7549). Post-hoc graphical analysis of residuals showed that the ANOVA model was fairly appropriate in both cases (Figure 3). Using the Tukey HSD test, significant differences were observed in mean values of FD100X and FD400X between groups of normal CG and epitheliomas as well as between normal CG and well or poorly differentiated carcinomas. Equally, significant differences were observed between CG adenomas and CG epitheliomas or well or poorly differentiated CG carcinomas. Detailed P-values between all individual groups (with significant values in red) are presented in Table 4. Value of Spearman correlation coefficient between the histological type of tumor (or normal CG) and values of FD100X was 0.659 , the same value for FD400X was 0.635 . Correlation between FD100X and FD400X and vimentin score was significant $(\mathrm{P}<0.000001)$ and high. The Pearson correlation was 0.868 (FD100X) and 0.863 (FD400X), while Spearman correlation was 0.881(FD100X) and 0.892 (FD400X). Figure 4 depicts the plot between these two values categorized according to their diagnoses.
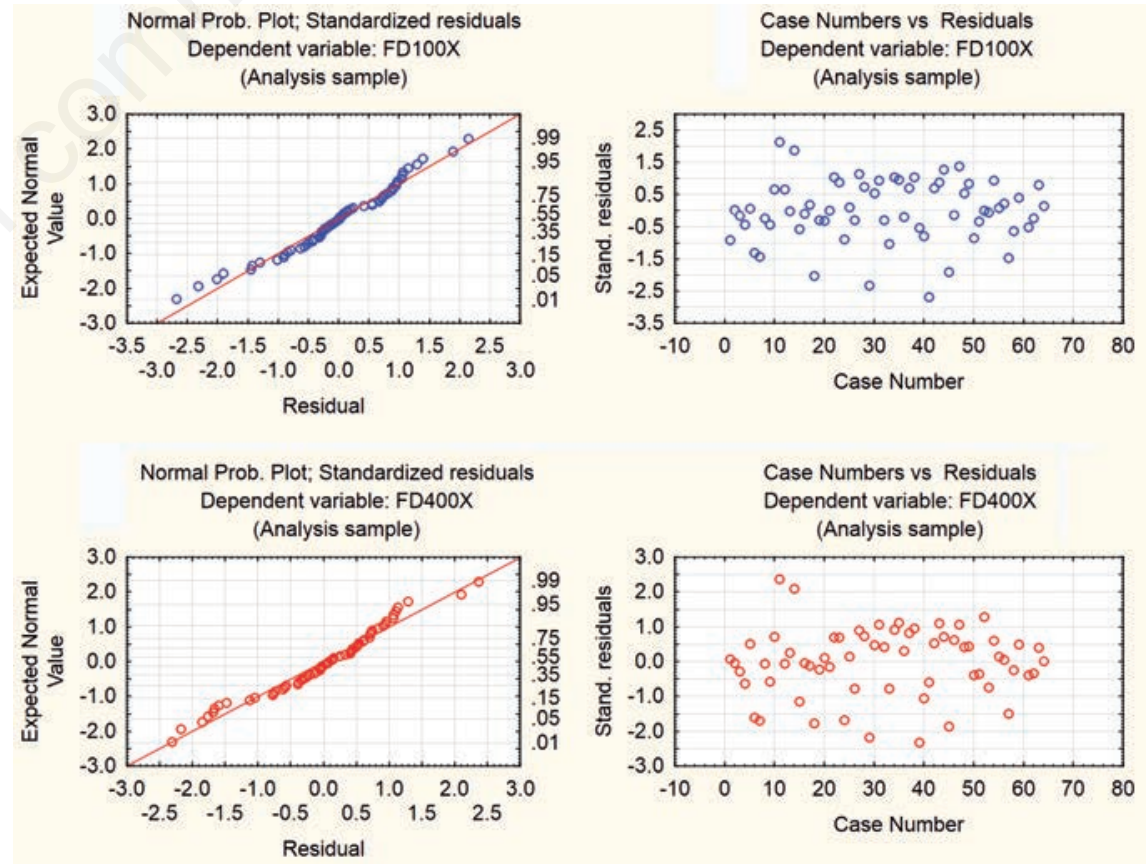

Figure 3. Analysis of residuals in ANOVA model. Normal probability plots and scatter plots of standardized residuals obtained by analysis of variance in FD 100X and FD 400X with respect to histological diagnosis as factor.

Case Numbers vs Residuals Dependent variable: FD100X (Analysis sample)

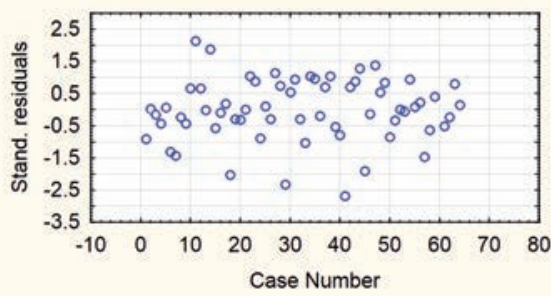
. 


\section{Discussion}

Our goal was to demonstrate the applicability and usefulness of a fractal geometry based method to differentiate between various histological types of tumors, and normal circumanal gland tissues in dogs. As our results show, this method yields significantly different, yet real number numerical results when comparing some of the investigated histological types of normal or neoplastic circumanal gland. Moreover, looking at Figure 2 it is evident that with the increase of malignancy in the histo- logical type of tumor there is also a corresponding increase in values of either FD100X or FD400X. This is also substantiated with the values of Spearman correlation coefficients between the histological type of tumor (or normal CG) and values of FD100X or FD400X, which were both above 0.600 .

Table 2. Vimentin score of samples included in the study.

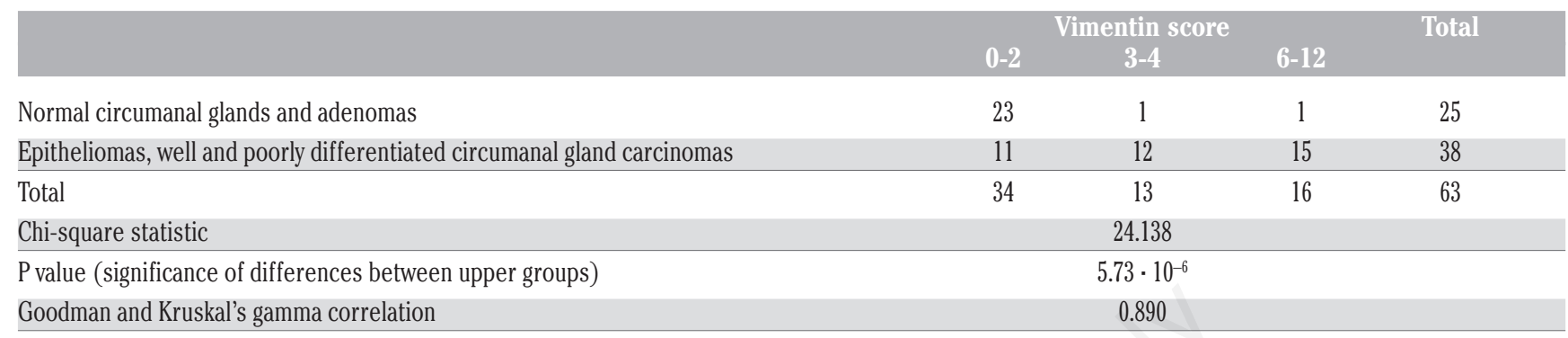

Data is consolidated into two groups of histological diagnosis and three groups of vimentin scores to render it suitable for the Chi square test (to calculate P-value), and Goodman and Kruskal's gamma statistics.

Table 3. Fractal dimension of normal circumanal glands and circumanal gland tumors.

\begin{tabular}{|c|c|c|c|c|c|c|}
\hline & & $\begin{array}{l}\text { Normal } \\
\text { circumanal } \\
\text { glands }\end{array}$ & $\begin{array}{l}\text { Circumanal } \\
\text { gland } \\
\text { adenomas }\end{array}$ & $\begin{array}{l}\text { Circumanal } \\
\text { gland } \\
\text { epitheliomas }\end{array}$ & $\begin{array}{l}\text { Well differentiated } \\
\text { circumanal gland } \\
\text { carcinomas }\end{array}$ & $\begin{array}{l}\text { Poorly differentiated } \\
\text { circumanal gland } \\
\text { carcinomas }\end{array}$ \\
\hline Mean value of FD & $\begin{array}{l}\text { FD100X } \\
\text { FD400X }\end{array}$ & $\begin{array}{l}1.318 \\
1.372\end{array}$ & $\begin{array}{l}1.384 \\
1.408\end{array}$ & $\begin{array}{l}1.547 \\
1.597\end{array}$ & $\begin{array}{l}1.569 \\
1.607\end{array}$ & $\begin{array}{l}1.679 \\
1.723\end{array}$ \\
\hline Lowest recorded value of FD & $\begin{array}{l}\text { FD100X } \\
\text { FD400X }\end{array}$ & $\begin{array}{l}1.138 \\
1.188\end{array}$ & $\begin{array}{l}1.208 \\
1.199\end{array}$ & $\begin{array}{l}1.216 \\
1.311\end{array}$ & $\begin{array}{l}1.281 \\
1.338\end{array}$ & $\begin{array}{l}1.600 \\
1.675\end{array}$ \\
\hline Highest recorded value of FD & $\begin{array}{l}\text { FD100X } \\
\text { FD400X }\end{array}$ & $\begin{array}{l}1.435 \\
1.531\end{array}$ & $\begin{array}{l}1.649 \\
1.701\end{array}$ & $\begin{array}{l}1.719 \\
1.733\end{array}$ & $\begin{array}{l}1.710 \\
1.746\end{array}$ & $\begin{array}{l}1.779 \\
1.785\end{array}$ \\
\hline Standard error of Mean FD & $\begin{array}{l}\text { FD100X } \\
\text { FD400X }\end{array}$ & $\begin{array}{l}0.029 \\
0.030\end{array}$ & $\begin{array}{l}0.032 \\
0.037\end{array}$ & $\begin{array}{l}0.050 \\
0.046\end{array}$ & $\begin{array}{l}0.026 \\
0.026\end{array}$ & $\begin{array}{l}0.028 \\
0.019\end{array}$ \\
\hline Std. deviation of FD & $\begin{array}{l}\text { FD100X } \\
\text { FD400X }\end{array}$ & $\begin{array}{l}0.092 \\
0.096\end{array}$ & $\begin{array}{l}0.125 \\
0.142\end{array}$ & $\begin{array}{l}0.167 \\
0.153\end{array}$ & $\begin{array}{l}0.120 \\
0.117\end{array}$ & $\begin{array}{l}0.069 \\
0.047\end{array}$ \\
\hline Mean measurement std. deviation & $\begin{array}{l}\text { FD100X } \\
\text { FD400X }\end{array}$ & $\begin{array}{l}0.049 \\
0.077\end{array}$ & $\begin{array}{l}0.062 \\
0.059\end{array}$ & $\begin{array}{l}0.058 \\
0.048\end{array}$ & $\begin{array}{l}0.071 \\
0.055 \\
\end{array}$ & $\begin{array}{l}0.046 \\
0.033\end{array}$ \\
\hline $\begin{array}{l}\text { Lilliefors' statistic } \\
\text { (P-value) } \\
\text { Shapiro-Wilk's statistic } \\
\text { (P-value) }\end{array}$ & $\begin{array}{l}\text { FD100X } \\
\text { FD400X } \\
\text { FD100X } \\
\text { FD400X }\end{array}$ & $\begin{array}{l}0.178(\mathrm{P}>0.20) \\
0.132(\mathrm{P}>0.20) \\
0.948(\mathrm{P}=0.64) \\
0.979(\mathrm{P}=0.96)\end{array}$ & $\begin{array}{l}0.207(\mathrm{P}<0.10) \\
0.146(\mathrm{P}>0.20) \\
0.920(\mathrm{P}=0.19) \\
0.930(\mathrm{P}=0.27)\end{array}$ & $\begin{array}{c}0.245(\mathrm{P}<0.10) \\
0.301^{* *}(\mathrm{P}<0.01) \\
0.884(\mathrm{P}=0.12) \\
0.820^{*}(\mathrm{P}=0.02)\end{array}$ & $\begin{array}{c}0.183 *(\mathrm{P}<0.05) \\
0.173(\mathrm{P}<0.10) \\
0.898^{*}(\mathrm{P}=0.03) \\
0.894^{*}(\mathrm{P}=0.03)\end{array}$ & $\begin{array}{l}0.159(\mathrm{P}>0.20) \\
0.230(\mathrm{P}>0.20) \\
0.955(\mathrm{P}=0.78) \\
0.876(\mathrm{P}=0.25)\end{array}$ \\
\hline
\end{tabular}

FD, fractal dimension; FD100X, fractal dimension estimated from vimentin IHC micrographs taken at magnification 100X; FD400X, fractal dimension estimated from vimentin IHC micrographs taken at magnification 400X; *significant at 5\%; ** significant at $1 \%$.

Table 4. P-values reflecting significant differences in fractal dimension between groups of non tumorous and tumorous circumanal glands.

$\begin{array}{lccccc}\text { FD400X } & \text { NCG } & \text { CGA } & \text { CGE } & \text { WDCGC } & \text { PDCGC } \\ \text { FD100X } & & 0.949743 & 0.001023 & 0.000189 & 0.000140 \\ \text { NCG } & 0.685997 & 0.002729 & 0.000251 & 0.000150 \\ \text { CGA } & 0.000843 & 0.012797 & & 0.999663 & 0.279804 \\ \text { CGE } & 0.000149 & 0.000507 & 0.989656 & & 0.265397 \\ \text { WDCGC } & 0.000138 & 0.000191 & 0.235540 & 0.318949 & \end{array}$

FD400X, fractal dimension estimated from vimentin IHC micrographs taken at magnification 400X; FD100X, fractal dimension estimated from vimentin IHC micrographs taken at magnification 100X; NCG, normal circumanal glands; CGA, circumanal gland adenomas; CGE, circumanal gland epitheliomas; WDCGC, well differentiated circumanal gland carcinomas; PDCGC, poorly differentiated circumanal gland carcinomas. Significant $\mathrm{P}$-values $(\mathrm{P}<0.05)$, determined by performing Tukey's HSD test, are in italic. 
At first it seems reasonable to think that the abovementioned differences are actually a mere consequence of differences in staining with vimentin (high Pearson and Spearman correlation between both of the FD-s and vimentin scores seem to point in that direction). Assuming that, one might think that this fractal analysis is redundant. We offer here few important reasons to discard this supposition. First, FD100X and FD 400X values provide numerical information about the complexity of the vimentin pattern found within normal tissue, tumor stroma, and cells. The values of FD100X and FD400X are read off the vimentin stain, and are therefore also dependent on it. However, in this case, vimentin is just a stain that visualizes the complexity within a tumor, which cannot be appreciated with the simple value of a vimentin score. Second, determining the score manually leaves a lot of space for subjectivity, which is reduced in our described method of determining FD100X or FD400X. Finally, values of FD are expressed as a very precise real number between 1.0 and 2.0. As such, these values are more attractive for comparison or any statistical analysis than values of a vimentin score, which can only be a whole number between 0 and 12 .

Nevertheless, it would be incorrect to dismiss the finding of different vimentin staining among various groups of normal and neoplastic CG. This is, without doubt, an important

Scatterplot of FD100X against Vimentin score $(0-12)$; categorized by Diagnosis

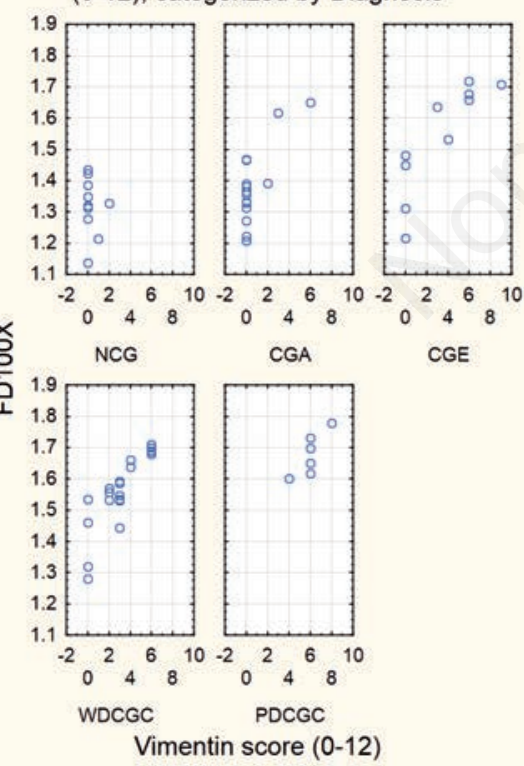

Scatterplot of FD400X against Vimentin score (0-12); categorized by Diagnosis

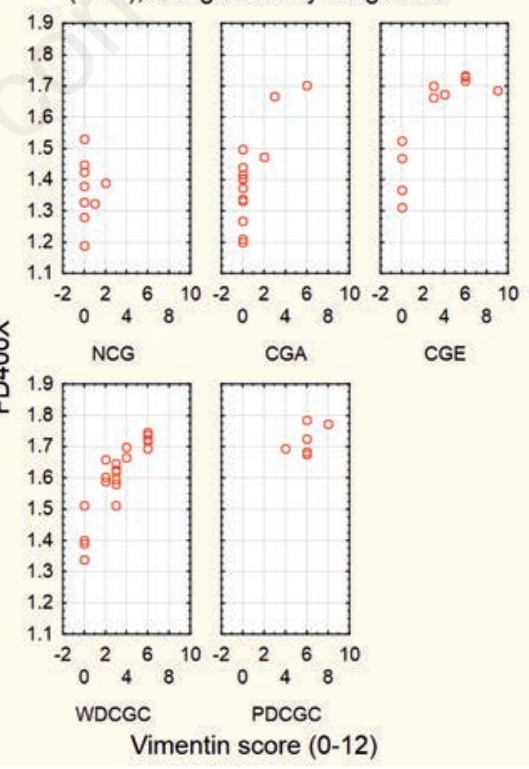

Figure 4. Scatter plots of FD100X and FD400X vs vimentin scores, categorized by histological diagnoses. On the left (blue dots) are represented values of FD100X vs vimentine scores, while on the right (red dots) are the values of FD400X vs vimentine scores. NCG, normal circumanal glands; CGA, circumanal gland adenomas; CGE, circumanal gland epitheliomas; WDCGC, well differentiated circumanal gland carcinomas; PDCGC, poorly differentiated circumanal gland carcinomas. observed in malignant epithelial (reserve and/or hepatoid) cells of poorly differentiated CG carcinomas is one of first steps in gaining the mesenchymal phenotype within EMT. The higher values of FD100X and FD400X observed in the more malignant histological subtypes of CG tumors is clearly a consequence of higher expression of vimentin, but also reflects the complex pattern of staining observed in these tumors. This complexity of staining pattern of vimentin within epitheliomas and carcinomas was a net result of two things: pronounced vimentin stain in the reserve cells, and intermingled distribution pattern of the same cells. These two factors produced vimentin stains with repeatedly similar patterns within same sample, and also at different magnifications. In our opinion, this observed pseudofractal and chaotic pattern of reserve cells within epitheliomas and the well and poorly differentiated carcinomas of CG was the main reason why FD100X and FD400X were so different between these semi-malignant or malignant types of tumors and benign tumors of CG (CG adenomas) or normal CG (Table 4). The positivity observed within hepatoid cells certainly altered the overall value of FD100X and FD400X, but in our opinion was not crucial. With the exception of normal CG and some adenomas, the same was true for the effect of positive staining of the fibrovascular stroma.

When strictly focusing on the comparison of the results of FD100X and FD400X it is evident that both values show equally good discriminating potential between various groups of normal or neoplastic CG. This is underscored by the very close p obtained by one-way ANOVA $\left(5.482 \cdot 10^{-8}\right.$ and $\left.3.750 \cdot 10^{-8}\right)$, and also by similar values of the Tukey HSD test. The latter is evident in Table 4 : both values succeeded to significantly discern between same groups of normal or neoplastic CG. It is therefore clear that both values are equally useful, and in a practical situation, only one would suffice. This finding also leads us to believe that microscopic magnification of such slides (at least within this given range) does not importantly affect the value of FD. This could very well be because of self similarity pattern of vimentin stained sections of neoplastic (or normal) CG at all magnifications (which could be granted by a self similarity of a bundle of vimentin filaments and a single filament at all dimensions).

We are aware of the weaknesses of the applied methodology. The first is the selection of the fields of view for the micrograph acquisition. One could avoid this potential subjectivity in choosing these fields if a digitization of the whole slide was conducted and such whole slide image was analyzed using a fractal algorithm. ${ }^{18,19}$ Alternatively, this problem could be addressed by choosing a few random parts of 
the tumor sample, ${ }^{13,14}$ which is in fact very similar to the solution we used (selection of three random fields of view). It may be argued that another flaw in the methodology is the fact that there was no real measure how much the positive signals from the tumor/circumanal gland stroma influenced overall values of FD100X and FD400X. In our opinion the complexity of fibrous tissue stroma followed the complexity of each individual tumor, this way enhancing the overall vimentin stain and values of FD100X and FD400X. However, we should point out here that in the case of normal CG, the connective tissue stroma was the only source of positive vimentin signals. Finally, a small portion of positive signals on vimentin stained sections was lost during the conversion to binary (bitmap) format of the images. However, all of the pictures were converted in the same way, so this affected all of the samples equally. As a final note, when addressing all possible limitations of the methodology used, we wish to state here that one of the expectations for the applied method was to be widely accessible, applicable, simple and inexpensive.

Despite all the potential pitfalls of this presented approach, we believe that this method could be useful as an orientative diagnostic test in discerning subtypes of CG tumors. This is especially true when discerning between CG adenomas and epitheliomas, or adenomas and carcinomas. Furthermore, this approach, which combines vimentin staining and fractal analysis, could be potentially used in other types of tissues/tumors, either as an indicator of complexity of tumor stroma, or as an indicator of complexity of positive signals within tumor cells. This is the first investigation in veterinary pathology which combines these two approaches (vimentin IHC and FD calculation), so we hope that future studies will improve our method and eventually integrate it in certain aspects of diagnostic routine.

In conclusion, vimentin staining and calculation of FD gives significantly different results between benign and malignant or semi-malignant types of CG tumors. These findings suggest that a more elaborate method founded on these principles would be helpful when discerning between all types of CG tumors. It also demonstrates the applicability of fractal based methods in veterinary oncology.

\section{References}

1. Goldsmith MH, Shofer FS. Skin tumors of the dog and the cat. Butterworth Heinemann, Oxford: 1992.

2. Lee Gross T, Ihrke PJ, Walder EJ, Affolter VK. Sebaceous tumors. In: Skin diseases of the dog and cat: clinical and histopathologic diagnosis, 2nd ed. Blackwell Science Ltd. Oxford: 2005, p. 641-664.

3. Goldschmidt, MH, Dunstan RW, Stannard AA, Von Tscharner C, Walder EJ, Yager JA. Histological classification of epithelial and melanocytic tumors of the skin of domestic animals. 3. Armed Forces Institute of Pathology, Washington DC: 1998.

4. Preziosi R, Della Salda L, Ricci A, Simoni P, Marcato PS. Quantification of nucleolar organiser regions in canine perianal gland tumors. Res Vet Sci 1995; 58:277-81.

5. Pereira RS, Schweigert A, Dias de Melo G, Vissani Fernandes F, Ruiz Sueiro FA, Fabrino Machado G. Ki-67 labeling in canine perianal glands neoplasms: a novel approach for immunohistological diagnostic and prognostic. BMC Vet Res 2013;9:83.

6. Lopes, R, Betrouni N. Fractal and multifractal analysis: a review. Med Image Anal 2009;13:634-46.

7. Iwabuchi M., Endoh M, Hiwatashi N, Kinouchi Y, Shimosegawa T, Masuda T, et al. Three-dimensional reconstruction and fractal geometric analysis of serrated adenoma. Jpn J Cancer Res 2002;93:259-66.

8. Delides A, Panayiotides I, Alegakis A, Kyroudi A, Banis C, Pavlaki A, et al. Fractal dimension as a prognostic factor for laryngeal carcinoma. Anticancer Res 2005;25: 2141-4.

9. Abu-Eid R, Landini G. Morphometrical differences between pseudo-epitheliomatous hyperplasia in granular cell tumours and squamous cell carcinomas. Histopathology 2006; 48:407-16.

10. Ahammer H, Helige C, Dohr G, WeissFuchs U, Juch H. Fractal dimension of the choriocarcinoma cell invasion front. Physica D 2008; 237:446-53.

11. Einstein, AJ, Wu HS, Sanchez M, Gil J. Fractal characterization of chromatin appearance for diagnosis in breast cytology. J Pathol 1998;185:366-81.

12. Goutzanis L, Papadogeorgakis N, Pavlopoulos PM, Katti K, Petsinis V, Plochoras I, et al. Nuclear fractal dimension as a prognostic factor in oral squamous cell carcinoma. Oral Oncol 2008;44:345-53.

13. Tambasco M, Magliocco AM. Relationship between tumor grade and computed architectural complexity in breast cancer specimens. Hum Pathol 2008;39:740-6.

14. Tambasco M, Eliasziw M, Magliocco AM. Morphologic complexity of epithelial architecture for predicting invasive breast cancer survival. J Transl Med 2010;8:140.

15. Lee LH, Tambasco M, Otsuka S, Wright A, Klimowicz A, Petrillo S, et al. Digital differentiation of non-small cell carcinomas of the lung by the fractal dimension of their epithelial architecture. Micron 2014;67: 125-31.

16. De Vico G, Cataldi M, Maiolino P, Carella F, Beltraminelli S, Losa GA. Fractal pattern of canine trichoblastoma. Anal Quant Cytol Histol 2011;33:151-7.

17. Sabo E, Boltenko A, Sova Y, Stein A, Kleinhaus S, Resnick MB. Microscopic analysis and significance of vascular architectural complexity in renal cell carcinoma. Clin Cancer Res 2001;7:533-7.

18. Di Ieva A, Grizzi F, Ceva-Grimaldi G, Russo C, Gaetani P, Aimar E, et al. Fractal dimension as a quantitator of the microvasculature of normal and adenomatous pituitary tissue. J Anat 2007;211:673-80.

19. Di Ieva A, Grizzi F, Sherif C, Matula C, Tschabitscher M. Angioarchitectural heterogeneity in human glioblastoma multiforme: a fractal-based histopathological assessment. Microvasc Res 2011;81:222-30.

20. Taverna G, Colombo P, Grizzi F, Franceschini B, Ceva-Grimaldi G, Seveso M, et al. Fractal analysis of two-dimensional vascularity in primary prostate cancer and surrounding non-tumoral parenchyma. Pathol Res Pract 2009;205:438-44.

21. Karslioğlu Y, Günal A, Kurt B, Ongürü 0, Ozcan A. Fractal dimension of microvasculature in renal oncocytomas and chromophobe renal cell carcinomas. Pathol Res Pract 2009; 205:677-681.

22. Fuseler, JW, Bedenbaugh A, Yekkala K, BaudinoTA. Fractal and image analysis of the microvasculature in normal intestinal submucosa and intestinal polyps in Apc(Min/+) mice. Microsc Microanal 2010;16:73-9.

23. Severin K, Hohšteter M, Artuković B, Gudan Kurilj A, Beck A, ŠoštarićZuckermann IC, et al. Fractal dimension of the hepatoid adenomas and carcinomas in dogs. Proc. 29th Meet. European Society of Veterinary Pathology and European College of Veterinary Pathologists, Uppsala, Sweden: 2011.

24. Burgess HJ, Kerr ME. Cytokeratin and vimentin co-expression in 21 canine primary pulmonary epithelial neoplasms. J Vet Diagn Invest 2009;21:815-20.

25. Theiler J. Estimating fractal dimension. J Opt Soc Am 1990;7:1055-73.

26. Baish JW, Jain RK. Fractals and cancer. Cancer res 2000;60:3683-8.

27. Sheskin DJ. Handbook of parametric and nonparametric statistical procedures, 2nd ed. Chapman Hall CRC, Boca Raton: 2002.

28. Ghasemi A, Zahediasl S. Normality tests for statistical analysis: a guide for non-statisticians. Int J Endocrinol Metab 2012; 10:486-9.

29. Montgomery DC. Design and analysis of experiments, 5 th ed. J. Wiley \& Sons, New 
York: 2001.

30. Vos JH, Van Den Ingh TS, Ramaekers FC, De Neijs M, Van Mil FN, Ivanyi D. Keratin and vimentin distribution patterns in the epithelial structures of the canine anal region. Anat Rec 1992;234:391-8.

31. Vos JH, Van Den Ingh TS, Ramaekers FC, Molenbeek RF, De Neijs M, Van Mil FN, et al. The expression of keratins, vimentin, neurofilament proteins, smooth muscle actin, neuron-specific enolase, and synaptophysin in tumors of the specific glands in the canine anal region. Vet Pathol 1993;30:352-61.

32. Jasik A, Kozaczinski W, Reichert M. Canine skin tumours with adnexal differ- entiation: histopathology and immunohistochemistry. Bull Vet Inst Pulawy 2009;53: 277-83.

33. Kalluri R, Weinberg RA. The basics of epithelial-mesenchymal transition. J Clin Invest 2009;119:1420-8. 